Case Study

\title{
CASE SERIES OF POTT'S SPINE DIAGNOSED BY ZN STAIN AND BACTEC MGIT IN A TERTIARY CARE HOSPITAL
}

\author{
Kumar $\mathbf{A}^{1}$, Das $\mathbf{P}^{2}$ \\ ${ }^{1}$ Consultant, Department of Microbiology, The Mission Hospital, Bidhanagar, Durgapur, India \\ ${ }^{2}$ Junior Consultant, Department of Microbiology, The Mission Hospital, Bidhanagar, Durgapur, India
}

\section{ABSTRACT}

Introduction: Pott's spine is a destructive form of tuberculosis and accounts for approximately half of all cases of musculoskeletal tuberculosis. Spinal Tuberculosis is most often missed due to inadequate sample and lack of clinical history. Most of the Extrapulmonary tuberculosis (EPTB) can be diagnosed by Ziehl Nelson stain (ZN) only, when clinically suspected samples are adequate and optimally stained. We are reporting four case series of spinal tuberculosis diagnosed by ZN stain and confirmed by Bactec MGIT Culture.

Material \& Methods: These four clinically suspected spinal biopsis were received for ZN stain and MTB culture by MGIT.

Results: All the four spinal biopsies were found positive by ZN stain as well as by BacTec MGIT.All the four cases were HIV negative.ESR and CRP of all four cases were raised.

Discussion \& Conclusion: Spinal tuberculosis can be easily diagnosed by ZN stain in resource constraint lab. Despite its common occurrence and the high frequency of long-term morbidity, there are no straight forward guidelines for the diagnosis and treatment of spinal tuberculosis. Early diagnosis and prompt treatment is necessary to prevent permanent neurological disability and to minimize spinal deformity.

\section{INTRODUCTION}

Pott's spine is a destructive form of tuberculosis and accounts for approximately half of all cases of musculoskeletal tuberculosis. Spinal Tuberculosis is, most often missed due to inadequate sample and lack of clinical history. Many of the (Extra pulmonary tuberculosis) EPTB can be diagnosed by Ziehl Nelson stain (ZN) only, when clinically suspected samples are adequate and optimally stained. The risk of developing tuberculosis is estimated to be 20-37 times greater in people co-infected with HIV than among those without HIV infection. ${ }^{.}$We are reporting four case series of spinal tuberculosis diagnosed by ZN stain and confirmed by Bactec MGIT Culture.

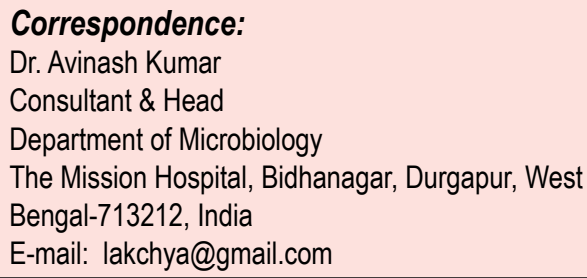

\section{CASE HISTORY}

A series of four cases with similar complaints of significant low back pain were admitted in our tertiary hospital.

\begin{tabular}{|l|l|l|l|l|}
\hline & Case1 & Case 2 & Case 3 & Case 4 \\
\hline Age & 50 yrs & 61 yrs & 40yrs & 30yrs \\
\hline Sex & Male & Male & Female & Female \\
\hline $\begin{array}{l}\text { Co mor- } \\
\text { bid condi- } \\
\text { tion }\end{array}$ & $\begin{array}{l}\text { Hyperten- } \\
\text { sion }\end{array}$ & $\begin{array}{l}\text { Type2 } \\
\text { Diabetes } \\
\text { mellitus }\end{array}$ & Nil & Nil \\
\hline $\begin{array}{l}\text { Site of } \\
\text { Lesion }\end{array}$ & $\begin{array}{l}\text { Lumbar } \\
\text { (L1-L3) }\end{array}$ & D8-D9 & D9-10 & $\begin{array}{l}\text { D11- } \\
\text { D12 }\end{array}$ \\
\hline
\end{tabular}

\section{MATERIAL MEHTODS}

All four cases were investigated radiologically and by our laboratory services to find out the etiology.

Biopsy of all cases were taken after all aseptic 
precautions from different site of lesion for Histopathology, ZN staining and Bactec MGIT Culture.

ZN stain was done with a positive control as per standard guidelines.

Complete investigations with different parameters were done to diagnose the cause of spinal tuberculosis.(Table-1).

\begin{tabular}{|c|c|c|c|c|}
\hline Parameters & Case1 & Case2 & Case3 & Case4 \\
\hline Total Count & 13100 & 11900 & 13200 & 16900 \\
\hline ESR & $70 \mathrm{MM} 1 \mathrm{hr}$ & $80 \mathrm{MM} 1 \mathrm{hr}$ & $72 \mathrm{~mm} 1 \mathrm{hr}$ & $18 \mathrm{~mm} 1 \mathrm{hr}$ \\
\hline CRP & 148.7 & 88.4 & 43.30 & 17.07 \\
\hline $\begin{array}{l}\text { Hemoglobin(g } \\
\mathrm{m} / \mathrm{dl}\end{array}$ & 8.4 & 8.8 & 8.5 & 10 \\
\hline $\begin{array}{l}\text { ELISA (Viral } \\
\text { markers) }\end{array}$ & Negative & Negative & Negative & Negative \\
\hline Procalcitonin & & 2.69 & & \\
\hline ZN Stain & $\begin{array}{l}\text { 18-20 AFB/100 Oil } \\
\text { Immersion Field }\end{array}$ & $\begin{array}{l}20-22 \\
\text { AFB/100 Oil } \\
\text { Immersion } \\
\text { Field }\end{array}$ & $\begin{array}{l}15-20 \\
\text { AFB/100 Oil } \\
\text { Immersion } \\
\text { Field }\end{array}$ & $\begin{array}{l}5-10 \text { AFB } / 100 \\
\text { Oil Immersion } \\
\text { Field }\end{array}$ \\
\hline Bactec MGIT & Positive & Positive & Positive & Positive \\
\hline
\end{tabular}

\section{RESULTS}

\section{Case1}

The ZN stain of spinal tissues shows plenty of neutrophils with 18-20 AFB/100 Oil Immersion Field (OIF) (Fig-1).

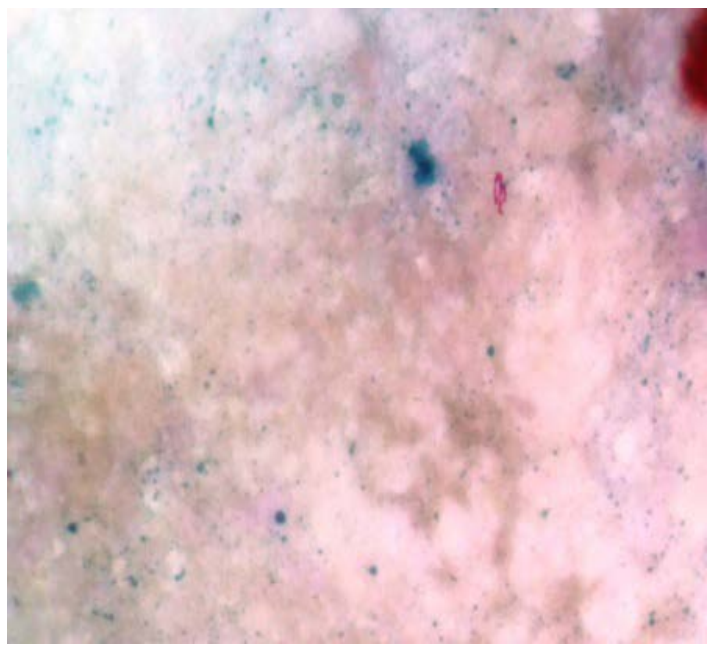

TB culture by Bactec MGIT was found to be positive. Histopathological findings showed granulomatous lesion suggestive of tuberculous origin and was negative for malignancy.
Radiological Findings: MRI Spine shows spondylodiscitis at L1-L3 level with introsseous abscess formation with extension of abscess in the ventral epidural space of spinal canal causing spinal canal stenosis along with Right psoas myositis and left psoas muscle abscess $(46.7 \times 28.4 \times 53.3 \mathrm{~mm})$. It also showed degenerative changes at L5-S1 level. (Fig-2)

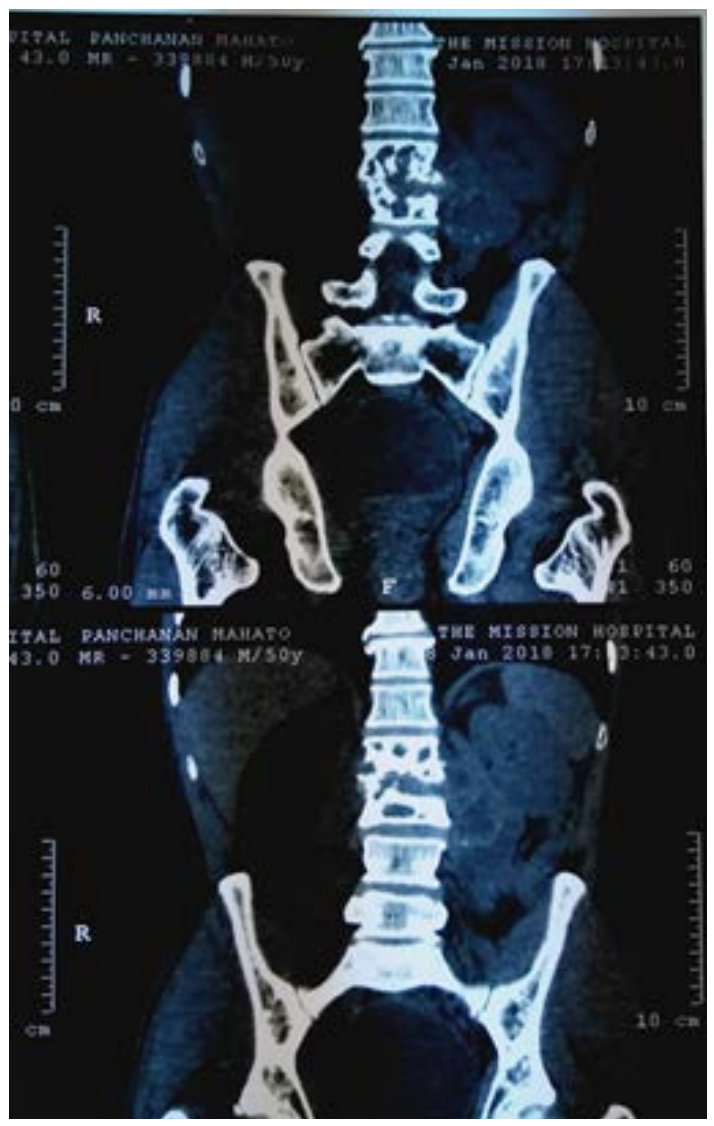

\section{Case2}

Biopsy from D8 -D9 was taken for Histopathology, ZN staining and Bactec MGIT Culture. The ZN stain of spinal tissues showed many neutrophils with 2022 AFB/100 OIF.TB culture with Bactec MGIT was positive for TB. Histopathological findings showed areas of necrosis, few epithelioid granulomas and many entrapped necrotic bony trabeculae. No malignancy was seen.

MRI dorsal Spine showed spondylodiscitis at D9D10 level with spondylitis involving D8, D9 \&D10 vertebral bodies. Paravertible and extradural collection behind D9 and D9-D10 disc with resultant spinal cord compression. Tuberculosis etiology was suspected due to calcified bodies in paravertible collection. 
CT scan of dorsal spine showed lytic destruction of D9 vertebrae with associated mild paraspinal collection and calcification.Narrowing of left D8-D9 neural foramina.

All lab reports were within normal range except ESR (80MM 1hr), Total count (11900), Hb (8.8), CRP(88.4), procalcitonin (2.69), CA 125(44.4) .

\section{Case3}

After all aseptic precaution, biopsy from D9-D10 were taken for Histopathology, ZN staining and Bactec MGIT Culture (Table-2). The ZN stain of spinal tissues shows plenty of neutrophil with 10 AFB/100 OIF .TB culture with Bactec MGIT was positive for MTB . Histopathological findings showed granulomatous lesion suggestive of tuberculous origin and it was negative for malignancy.

All lab reports were within normal range except ESR (72MM 1hr), Total count (13200), Hb (8.5), CRP (43.30) .

MRI dorsal Spine showed infective spondylitis at D9-D10 level with destruction of intervertebral disc and adjacent vertebral end plates seen.D9 vertebra was completely destroyed. Spondylitis was seen at D6, D7, D8 and D11.Abscess was seen at D9-D10 disc at endplate level with extension of abscess in the pre and para vertebral region, in the ventral epidural space and at neural foramines. Pre and para vertebral abscess was seen from the level of superior end plate of D6 vertebra to D10 level. Intraosseous extension of collection seen in D7,D8 vertebral body with erosion of anterior cortex. Ventral epidural collection was seen posterior to D9 and D10 vertebral body.AtD7 level paravertebral collection was extending into adjacent pleural space and lung with consolidation.

\section{Case4}

Biopsy was taken from D11-D12 for Histopathology, ZN staining and Bactec MGIT Culture (Table-2). Plenty of neutrophil with $05 \mathrm{AFB} / 100$ OIF was seen by ZN stain of spinal tissues. TB culture with Bactec MGIT was positive for MTB.On Histopathological examination showed areas of necrosis, few epithelioid granulomas and many entrapped necrotic bony trabeculae.

All lab reports were within normal range except ESR (18 mm 1hr), Total count (16900), Hb (10), CRP (17.07).
MRI of dorsolumber spine: Spondylodiscitis noted at the level of D11-D12 causing kyphotic deformity and narrowing of spinal canal to approximately $6.4 \mathrm{~mm}$.Stretching of spinal cord at this level with altered signal intensity within suggestive of myelopathic changes.Pravertebral abscess noted along D11-D12 vertebra tracking along right ileopsoas muscle and forming a collection within right quadrates lumborum muscle at the level of 2 L3 vertebra measuring approximately $38 \times 45 \times 21$ mm. (Fig-3)

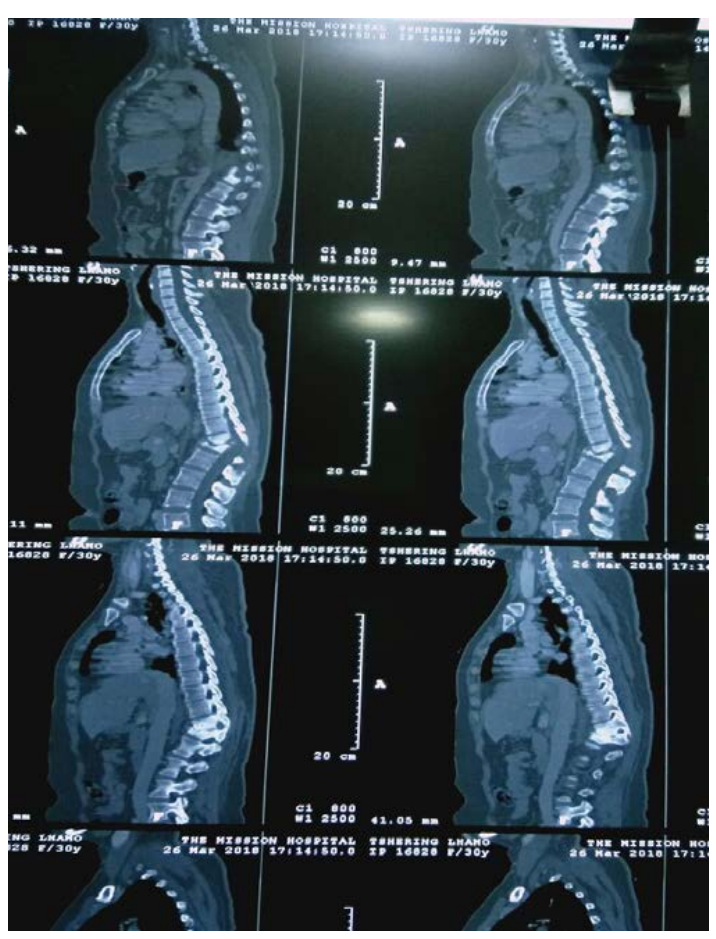

\section{DISCUSSION AND CONCLUSION}

Extrapulmonary TB (EPTB) constitutes about $15 \%-20 \%$ of all cases of TB. ${ }^{(2,3)}$ The diagnosis of Pott's spine is difficult due to the paucibacillary nature of sample, difficulty in obtaining specimens from deep-seated organs and inability to get an additional specimen. As an outcome, failure to diagnose and treat affected patients leading to increased morbidity and mortality, development of secondary resistance (including extensively drugresistant TB) and ongoing transmission of disease. ${ }^{(4)}$

In our case series, thoracic region is predominantly involved for spinal tuberculosis.

The prognosis for spinal tuberculosis can be improved by early diagnosis and rapid intervention. Spinal tuberculosis can be easily diagnosed by ZN stain in resource constraint lab with only little effort. 
Early diagnosis and prompt treatment is necessary to prevent permanent neurological disability and to minimize spinal deformity. All four patients were started with Anti tuberculosis treatment and they are doing extremely well.

\section{REFERENCES}

1. J ain AK. Tuberculosis of the spine: a fresh look at an old disease. J Bone Joint Surg Br2010; 92(7):905-13.
2. Schirmer $P$, Renault $C A$, Holodniy M. Is spinal tuberculosis contagious? Int J Infect Dis2010; 14(8):e659-66.

3. Sharma SK, Mohan A. Extrapulmonary tuberculosis. Indian J Med Res 2004; 120:316-53.

4. Iram S, Zeenat A, Hussain S, Wasim Yusuf N, Aslam M. Rapid diagnosis of tuberculosis using Xpert MTB/RIF assay - Report from a developing country. Pak J Med Sci 2015; 31:105-10. 